\title{
Neuropsychological Performance in Alcohol Dependent Patients: A One-Year Longitudinal Study
}

\author{
Lucia loime ${ }^{1}$, Riccardo Guglielmo ${ }^{2} 凶$, Giuseppe Fertonani Affini ${ }^{3}$, Marianna Quatrale ${ }^{2}$, \\ Giovanni Martinotti ${ }^{4}$, Antonino Callea ${ }^{1}$, Evelina Savi ${ }^{3}$, and Luigi Janiri ${ }^{2}$ \\ ${ }^{1}$ Department of Human Science, Lumsa University of Rome, Rome, Italy \\ ${ }^{2}$ Department of Geriatrics, Neuroscience and Orthopedics, Institute of Psychiatry, Catholic University Medical School of Rome, Rome, Italy \\ ${ }^{3}$ Department of Mental Health and Addictions, Local Health Unit of Parma, Parma, Italy \\ ${ }^{4}$ Department of Neuroscience and Imaging, University "G. D'Annunzio", Chieti, Italy
}

Objective Despite several studies that have highlighted the harmful effects of alcohol consumption on cognitive functions it remains unclear whether certain brain areas are more sensitive than others are or whether alcohol causes widespread cognitive deficit. Moreover, the role of continued abstinence has yet to be clarified regarding the quality of recovery on the different cognitive domains. The aim of this 1-year longitudinal study was to evaluate the recovery of cognitive deficits in the medium (6 months) and long term (12 months) after the interruption of drinking.

Methods Forty-one alcohol-dependent patients were recruited from two outpatient treatment facilities and cognitive functions were compared on a control group of forty healthy controls. The patients were then re-assessed at 6 and 12 months. Changes in neuropsychological measures were evaluated with repeated measures analysis of variance (ANOVA). We also compared 1-year follow-up scores with control data (unpaired t tests) to identify tests on which significant differences persisted.

Results Patients performed significantly worse than controls in all cognitive domains investigated and this cognitive impairment was evident in recently abstinent patients. A year of abstinence resulted in a significant improvement in all cognitive domains assessed after detoxification from alcohol. After year 1, alcoholic subjects had returned to normal levels compared to healthy controls on all domains except for general non-verbal intelligence, verbal memory and some visuospatial skills.

Conclusion Our results support the hypothesis of widespread impairment resulting from alcohol consumption. The recovery of cognitive functions is not homogeneous during prolonged abstinence.

Psychiatry Investig 2018;15(5):505-513

Key Words Alcohol, Cognition, Longitudinal.

\section{INTRODUCTION}

The neuropsychological assessment of alcoholic patients assumes a strategic importance both on the purely cognitive side, to plan strategies for psychosocial reintegration, and as an indicator of remission, for example through a recovery of the regulatory/modulator action of the prefrontal cortex on the subcortical systems. In fact, brain executive control networks are weakened or "tuned down" and appetitive drive

Received: July 12, 2017 Revised: September 20, 2017

Accepted: September 27, 2017

$\triangle$ Correspondence: Riccardo Guglielmo, MD, PhD

Department of Geriatrics, Neuroscience and Orthopedics, Institute of Psychiatry, Catholic University Medical School of Rome, Largo “A. Gemelli” 1, Rome, Italy

Tel: +39 3883723606, Fax: +39 0630157266, E-mail: ricguglielmo@gmail.com

(a) This is an Open Access article distributed under the terms of the Creative Commons Attribution Non-Commercial License (http://creativecommons.org/licenses/bync/4.0) which permits unrestricted non-commercial use, distribution, and reproduction in any medium, provided the original work is properly cited. networks are strengthened or "tuned up" in active alcoholism. ${ }^{1}$ The complicated mental mechanisms that regulate cognitive, emotional-affective and relational life aspects of an individual are completely subverted by the interaction with alcohol. The neuropsychological assessment throughout the diagnostic process of mental illness is very important. It allows procurement of a complete picture of the patient's cognitive efficiency, the timely identification of any underlying neurological diseases and the development of rehabilitation cognitive treatment, verification of the progress in the short and long term. Furthermore, it is also essential in the planning of appropriate therapeutic interventions that take into account the resources and the cognitive weaknesses of the subject. ${ }^{2-4}$ However, the literature is unanimous in considering that the cognitive impairment reduces the effectiveness of psychological treatments. ${ }^{5,6}$ Following neuropsychological assessment, paths can be taken to strengthen or recover cognitive functions affected 
by alcoholism. In fact, the neuronal synapses can change throughout life and some impaired neuropsychological functions, such as memory or attention may be recovered or improved through specific training alongside sustained abstinence. Moreover, some fMRI studies showed that abstinence maintenance is associated with compensatory changes in synchrony of some cerebral networks. In particular, abstinence has been associated with a greater synchrony of the executive control network and with a reduced synchrony of the appetitive drive network and this may facilitate the behavioral control required to maintain abstinence. ${ }^{1,7}$

Our work fits into the large field of research on the nature and quality of the cognitive deficits in alcoholics. There is already some evidence suggesting that alcoholics show cognitive deficits compared to non-alcoholics but conflicting evidence has emerged about the nature of cognitive deficits in relation to the different brain areas involved.

Some studies show a major involvement of the frontal areas, ${ }^{8-12}$ other evidence instead emphasizes on a greater degree of right hemisphere lateralization, ${ }^{13-16}$ finally, other studies have found a widespread deficit. ${ }^{17-19}$ In addition, the controversial question of the possibility of recovery of the cognitive functions over time remains. The majority of evidence shows that the recovery of the different cognitive functions depends on the type of function, some functions are recovered after few weeks, for others, however the deficit remains more stable over time..$^{13}$ Nevertheless, few studies monitor the evolution of cognitive functions on the same sample of alcoholics from the baseline up to a year using a comprehensive neuropsychological battery.

The main objective of our study is to evaluate the presence or absence of cognitive impairment in abstinent alcoholic patients compared to non-alcoholic subjects by using a comprehensive neuropsychological battery. Moreover, we have assessed the same sample of alcoholics at different times during the course of one year to study the changes in cognitive functioning. In this way it has been possible to highlight the difference in the nature and quality of cognitive deficits in the short ( $<1$ month), medium (6 months) and long term (12 months) from the interruption of drinking.

\section{METHODS}

\section{Participants}

We enrolled 41 patients ( 31 males, 10 females) (Figire 1) with a diagnosis of alcohol dependence according to DSM-

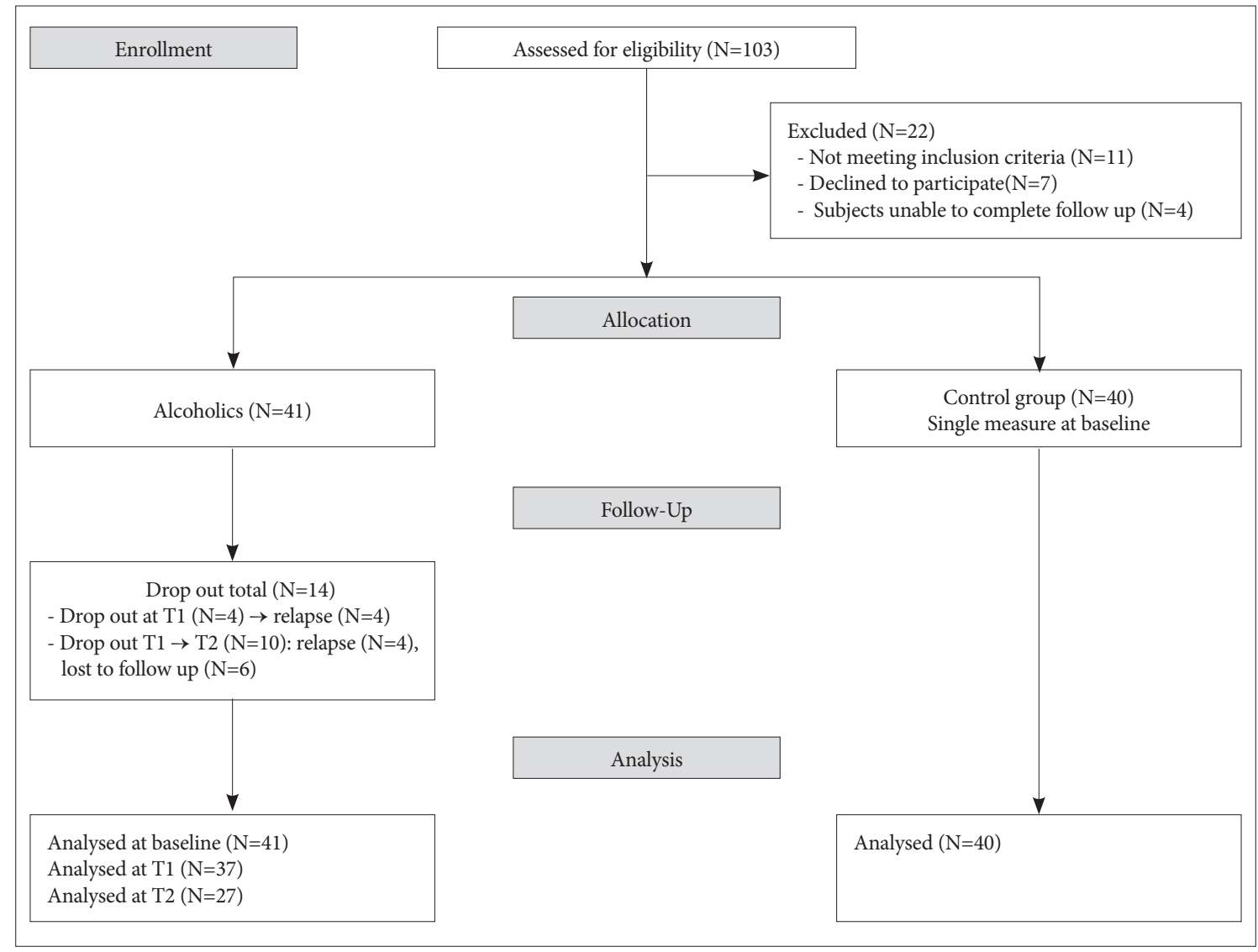

Figure 1. Flow diagram of patients and controls. T1: 6 months, T2: 1 year. 
IV-TR. ${ }^{20}$ The patients were recruited from outpatients programs at University General Hospital 'A. Gemelli' in Rome and at the outpatient alcohol unit of the drug addiction service of Parma. They were followed for one year, which included a baseline and follow-up 6 and 12 months later, each with comprehensive substance use and neuropsychological assessments administered by a trained Master's level research assistant. All the patients and controls were evaluated by attending psychiatrists using the Structured Clinical Interviews for DSM-IV (SCID I). ${ }^{21}$ Inclusion criteria were: age, between 18 and 65 years; Italian native speakers and diagnosed with alcohol dependence according to DSM-IV-TR. ${ }^{20}$ Patients were excluded if they had ever met DSM-IV-TR ${ }^{20}$ criteria for schizophrenia, mood disorders, caffeine addiction and substance abuse other than alcohol and nicotine; if they had a history of liver disease, medical or neurological illness, or trauma; if they had suffered a head injury involving loss of consciousness for more than $10 \mathrm{~min}$, or if they were currently taking medications, other than those approved for alcohol dependence, or illicit drugs that would affect the central nervous system (CNS).

The control group consisted of 40 healthy controls (28 males, 12 females) (Figure 1) recruited among the general population. The healthy controls subjects had no alcohol-related problems based on information obtained from semistructured interviews, questionnaires and biological alcoholism markers [e.g. Carbohydrate-deficient Transferrin (CDT)]. They were excluded if they had a history of medical or neurological illness or trauma that could affect the CNS; had ever met either DSM-IV-TR ${ }^{20}$ criteria for a major psychiatric disorder, including substance dependence or substance abuse; or had reported a period of time lasting more than 1 month when they had drank more than two standard drinks each day.

The study was approved by the Institutional Review Board and national regulatory authorities in accordance with local requirements and was conducted in accordance with Good Clinical Practice Guidelines and the Declaration of Helsinki (1964) and subsequent revisions (D.3.2.764.14). After receiving information on the intervention, all subjects provided written informed consent.

\section{Study procedures}

At enrolment, subjects who were currently drinking, signed an informed consent and then underwent detoxification for a period of 5 to 10 days, according to a validated protocol. ${ }^{22}$ After detoxification, patients started an anti-craving therapy with medication approved for alcohol dependence. ${ }^{23}$ Throughout the entire study period, they were monitored by psychiatrist on a monthly basis.
Patients were assessed 1 week after detoxification (end of benzodiazepine use) and prior to starting the anti-craving therapy (T0), after 6 (T1) and 12 months (T2). Healthy control subjects were assessed once. For all patients as well as healthy controls, the assessment comprised questionnaires on demographic, psychopathological and drinking-related variables. Information on past and recent alcohol consumption was obtained from the Time Line Follow Back Interview (TLFB) ${ }^{24}$ Clinical Global Impressions Scale Severity (CGI-S) ${ }^{25}$ was used to assess the severity of psychopathology, alcohol craving was evaluated using the Italian version of the Obsessive-Compulsive Drinking Scale (OCDS) ${ }^{26}$ and the Hamilton Depression Rating Scale (HAMD) ${ }^{27}$ was used to assess depressive symptoms. The duration of dependence, defined as the number of years from the diagnosis of alcohol dependence was made for the first time, and the age of onset of alcoholism were also assessed.

For both patients and control subjects, the battery for the measurement of cognitive functions was carried out in a single administration. The test session lasted about 1 hour and 30 minutes. A Master's level research assistant conducted all testing in a well-lit and soundproof room. In order to avoid a possible learning effect due to the repetition of assessments, parallel forms were used if provided by the test.

Abstinence from alcohol was determined based on selfevaluation measures and a family member interview. Abstinence was also confirmed by performing blood alcohol tests on each outpatient follow-up visit by measuring alcohol abuse indices [aspartate aminotransferase (AST), alanine aminotransferase (ALT), $\gamma$-glutamyl tranpeptidase (GGT), carbohydrate-deficient transferrin (CDT)]. Participants underwent urine toxicology prior to cognitive assessments to confirm abstinence from alcohol and/or other substance at each time point and encourage abstinence from substances to avoid capturing the acute adverse effects of recent use. Relapse was defined as the intake of any amount of alcohol. In the case of alcoholic relapse, subjects were excluded from the study (dropout), but continued to receive treatment.

\section{Neuropsychological Battery}

\section{Raven's progressive matrices 1938 (PM38)}

The PM38 assesses abstract reasoning and is regarded as a non-verbal estimate of fluid intelligence. ${ }^{28}$ This version of PM38 includes four sets (A, B, C, and D) of 12 black and white patterns that have to be completed from a multiple choice set of alternatives and items progressively increase in level of difficulty within and across sets. The subject had no time limit to complete the test, and the final score was calculated based on the accuracy of the performance (i.e., the number of correct answers). 


\section{Trail Making Test (TMT)}

The TMT is a measure of visual-conceptual and visual motor tracking skills, which focuses on divided attention, the ability to shift and mental flexibility. ${ }^{29}$ The test is given in two parts. Part A requires subjects to connect a series of consecutively numbered circles, Part B requires subjects to connect a series of numbered and lettered circles, alternating between the two sequences. The time difference (B-A) is also considered as reflecting cognitive activity and shifting ability. The score is the total time (in seconds) required to complete the task.

\section{Stroop colour word test (Stroop test)}

The Stroop Test is a neuropsychological tools used to asses selective attention, cognitive flexibility and sensitivity to interference. ${ }^{30}$ The Stroop effect was evaluated by computing a time interference effect (based on executive time) and an error interference effect (based on number of errors).

\section{The Modified Card Sorting Test (MCST)}

The MCST is a shortened version of the Wisconsin Card Sorting Test and is widely used in clinical settings for the evaluation of executive functions particularly the abstract reasoning skills and cognitive flexibility. ${ }^{31}$ Performance on the MCST was scored by computing the number of categories achieved by a participant, and the number of perseverative errors.

\section{Rey Auditory Verbal Learning Test (RAVLT)}

The RAVLT was used to provide a measure of verbal memory function..$^{32} \mathrm{~A}$ list of 20 words is read to the subject (one word every $2 \mathrm{~s}$ ). Afterwards the subject was asked to repeat as many words as he or she could remember (immediate recall-IR). After the immediate recall was finished, the list was repeated once after a fifteen minute interval and the subject was again required to recall the words he/she remember (delayed recall-DR). This test provides four parallel forms to limit the learning effect of repeated assessment.

\section{Rey-Osterrieth complex figure (ROCF)}

The ROCF consists of the direct copying of a complex bi dimensional figure and of its recollection from memory after a given delay (ten minutes). It is a useful tool to analyse the integrity of non-verbal memory, visuospatial abilities, planning, and perceptual, motor and Visio-constructional functions. ${ }^{33}$

\section{Test Copy of freehand drawings (Copy of Figures)}

This test is a sub-test of the Mental Deterioration Battery and it is used to assess the praxic constructive and graph-motor abilities. ${ }^{34}$ It consists in the copy of two simple geometric images (a cube and a star) and a stylized image (one house) of increasing difficulty.

\section{Statistical analysis}

T-tests (two-sided) and chi-square analyses using Fisher's exact test were used to examine differences between patients and controls, with respect to demographic variables.

In order to investigate differences in cognitive abilities between control and alcoholic group we performed t-tests for independent samples, considering significant differences for $\mathrm{p}<0.05$.

In order to investigate if alcohol abstinence reduces craving scores and improve cognitive performance we also performed a one-group repeated measures ANOVA for the 27 alcoholics for whom initial, 6-months, and 1-year scores were available. For the subtests with significant differences in the time, a post hoc test was carried out. We choose Tukey's test because it was designed for a situation with equal sample sizes, including repeated measures analysis. Furthermore, we calculated a measure of effect size for group mean differences through $\eta^{2}$. It can be interpreted using the Cohen's guidelines, ${ }^{35}$ i.e. small whether $\eta^{2}$ is between 0.01 and 0.05 , medium whether $\eta^{2}$ is between 0.06 and 0.13 large whether $\eta^{2}$ is higher than 0.14 .

We also performed t-tests for independent samples in order to compare 6-months and 1-year follow-up scores with control data in order to identify abilities on which significant differences still persisted.

Finally, to examine the potential effects of psychopathological severity on initial performance, we correlated CGI-S and HAMD scores with cognitive performance subtests related to verbal memory, visuospatial abilities and executive functions. We used Spearman correlation tests as the samples size was small.

\section{RESULTS}

\section{Comparison of alcohol-dependent patients and healthy controls at baseline}

Table 1 shows the sample characteristics for alcohol-dependent patients and for healthy controls. The groups were well matched for age, gender, education, employment and smoking status. As expected, we found a significantly higher score on the HAMD [t(79)=2,328, $\mathrm{p}<0.05]$. Table 2 shows the baseline clinical features of alcohol dependent patients. Of note, between the alcohol-dependent patients we have found a difference by gender at baseline in terms of cognitive function: for the Stroop errors interference t(39)=-2.07, $\mathrm{p}<$ 0.05 with women that had a significantly higher scores than men.

In order to investigate differences in verbal memory, visuospatial abilities and executive functions between control $(n=40)$ and alcoholic group $(n=41)$ we performed t-tests for independent samples (Table 3). 
Table 1. Demographic and clinical characteristics of alcohol-dependent patients and healthy controls

\begin{tabular}{|c|c|c|c|}
\hline & Alcoholics $(\mathrm{N}=41)$ & Controls $(\mathrm{N}=40)$ & Level of significance \\
\hline Women, N (\%) & $10(25)$ & $12(30)$ & NS \\
\hline Men, N (\%) & $31(75)$ & $28(70)$ & NS \\
\hline Age (years), mean (SD) & $46.63(8.5)$ & $46.60(6.2)$ & NS \\
\hline Education (years), mean (SD) & $11.8(4)$ & $13.2(3.4)$ & NS \\
\hline Subjects employed, N (\%) & $30(73.2)$ & $35(87.5)$ & NS \\
\hline HAMD, mean (SD) & $8.27(3.5)$ & $6.65(2.68)$ & $\mathrm{p}<0.05$ \\
\hline Smokers, N (\%) & $31(75.6)$ & $25(62.5)$ & NS \\
\hline CDT, \% (SD) & $2.6(3.5)$ & $1.1(1.8)$ & $\mathrm{p}<0.05$ \\
\hline
\end{tabular}

NS: not statistically significant group difference, CDT: Serum Carbohydrate Deficient Transferrin percentage (\%CDT), HAMD: Hamilton Rating Scale for Depression, SD: standard deviation

Table 2. Baseline alcohol addiction-related characteristics of alcohol-dependent patients

\begin{tabular}{lc}
\hline \multicolumn{1}{c}{ Clinical features } & Alcoholics (N=41) \\
\hline Age of onset, regular alcohol use, mean (SD) & $31.8(10.4)$ \\
$\begin{array}{l}\text { Duration of alcohol dependence (years), } \\
\text { mean (SD) }\end{array}$ & $13.82(9.96)$ \\
Number of standard drinks per day & $8(5.3)$ \\
in the previous 28 days, mean (SD) & \\
OCDS obsessive, mean (SD) & $6.73(4.2)$ \\
OCDS compulsive, mean (SD) & $9.34(4.4)$ \\
CGI_S, mean (SD) & $3.34(1.4)$ \\
\hline
\end{tabular}

OCDS: Obsessive-Compulsive Drinking Scale, CGI_S: Clinical Global Impressions Scale Severity, 1 standard drink: $12 \mathrm{~g}$ ethanol. SD: standard deviation

Alcoholic group had significantly lower scores than control group in RAVLT-IR [t(79) $=-4.664, \mathrm{p}<0.01]$ and RAVLTDR $[t(79)=-2.914, p<0.01]$, showing lower performance in verbal memory. Furthermore, alcoholic group had significantly lower scores than control group in ROCF-IR [ $t(79)=$ 3.330, $\mathrm{p}<0.01]$ and ROCF-DR [t(79)=-5.066, $\mathrm{p}<0.01]$, PM$38[\mathrm{t}(79)=-5.298, \mathrm{p}<0.01]$ and in Copy of Figures $[\mathrm{t}(79)=-2.478$, $\mathrm{p}<0.01$ ], showing lower performance in visuospatial abilities. Finally, alcoholic group had significantly higher scores than control group in MCST number of perseverative errors $[t(79)=$ $2.010, \mathrm{p}<0.05]$, STROOP colour word test (error interference effect) $[\mathrm{t}(79)=1.920, \mathrm{p}<0.05]$, STROOP colour word test (time interference effect) $[\mathrm{t}(79)=2.837, \mathrm{p}<0.01]$, TMT Part $\mathrm{B}$ $[\mathrm{t}(79)=2.521, \mathrm{p}<0.01]$ and TMT Part $\mathrm{B}-\mathrm{A}[\mathrm{t}(79)=2.952, \mathrm{p}<0.01]$, showing lower performance in executive functions while there was no difference in MCST number of categories $[t(79)=$ $-1.301, \mathrm{p}=0.19]$.

Results of Spearman correlation tests showed verbal memory subtests were not significantly related to psychopathological severity. Some subtests of visuospatial abilities were significantly related to psychopathological severity; in particular, ROCF-IR, ROCF-DR, and Copy of Figures were negatively related to CGI-S. Finally, with respect to executive functions, only MCST numbers of perseverative errors were significantly and positively related to CGI-S. HAMD scores and smoking status were not significantly correlated with any of the cognitive variables.

\section{Alcoholic Performance at Initial Testing and throughout 1-year follow-up}

In order to test the effects of alcohol abstinence on craving scores and cognitive performance a series of repeated measures ANOVA, between $\mathrm{T} 0, \mathrm{~T} 1$, and $\mathrm{T} 2$ were performed (Table 4). At 1-year follow-up (T2) the rate of drop-out was 35\% (14 subjects), so we performed this analysis on 27 subjects [gender ratio: 10 (37\%) women and 17 (63\%) men]. By means of $\mathrm{t}$-tests for independent samples we have found that there were no significant differences between drop-outs $(n=14)$ and no drop-outs $(\mathrm{n}=27)$ respect to demographic, clinical and neuropsychological data.

All subtests were significant with $\mathrm{p}<0.05$. With respect to craving, Tukey's post hoc test showed that obsessive and compulsive craving significantly decreased both between $\mathrm{T} 0$ and $\mathrm{T} 1$ and T1 and T2. With respect to verbal memory, Tukey's post hoc test showed that RAVLT-IR significantly increased between $\mathrm{T} 0$ and $\mathrm{T} 1$, but any significant difference was found between $\mathrm{T} 1$ and $\mathrm{T} 2$; while RAVLT-DR firstly significantly decreased from T0 to T1 (worse performance) and after significantly increased from T1 to T2 (better performance).

All subtest scores of visuospatial abilities significantly increased both between $\mathrm{T} 0$ and $\mathrm{T} 1$ and $\mathrm{T} 1$ and $\mathrm{T} 2$ as revealed by Tukey's post hoc test. The performance at executive functions significantly improved over time. In particular, Tukey's post hoc test for MCST number of categories significantly increased between $\mathrm{T} 0$ and $\mathrm{T} 1$, but any significant difference was found between T1 and T2; MCST number of perseverative errors significantly decreased between $\mathrm{T} 0$ and $\mathrm{T} 1$, but any significant difference was found between $\mathrm{T} 1$ and $\mathrm{T} 2$; finally, STROOP colour word test (error interference effect), STROOP colour word test (time interference effect),TMT Part 
Table 3. t-tests between control and alcoholic group at baseline

\begin{tabular}{|c|c|c|}
\hline & Alcoholics $(\mathrm{N}=41)$ & Controls $(\mathrm{N}=40)$ \\
\hline \multicolumn{3}{|l|}{ Verbal memory } \\
\hline RAVLT immediate recall, mean (SD) ${ }^{\dagger}$ & $40.42(9.76)$ & $49.83(8.33)$ \\
\hline RAVLT delayed recall, mean $(\mathrm{SD})^{\dagger}$ & $8.37(3.02)$ & $10.10(2.25)$ \\
\hline \multicolumn{3}{|l|}{ Visuospatial abilities } \\
\hline ROCF immediate recall, mean (SD) ${ }^{\dagger}$ & $27.81(6.42)$ & $31.33(1.87)$ \\
\hline ROCF delayed recall, mean $(\mathrm{SD})^{\dagger}$ & $9.47(6.54)$ & $16.47(5.87)$ \\
\hline PM38, mean $(\mathrm{SD})^{\dagger}$ & $31.92(10.02)$ & $40.91(3.89)$ \\
\hline Copy of Figures, mean (SD) ${ }^{\dagger}$ & $8.12(2.64)$ & $9.29(1.42)$ \\
\hline \multicolumn{3}{|l|}{ Executive functions } \\
\hline MCST categories, mean (SD) & $4.73(1.67)$ & $5.18(1.38)$ \\
\hline MCST perseverative errors, mean (SD)* & $4.33(6.20)$ & $2.09(3.40)$ \\
\hline STROOP errors interference, mean (SD)* & $1.54(2.63)$ & $0.73(0.50)$ \\
\hline STROOP time interference, mean $(\mathrm{SD})^{\dagger}$ & $23.95(9.60)$ & $19.02(5.42)$ \\
\hline TMT Part B, mean $(\mathrm{SD})^{\dagger}$ & $116.90(46.45)$ & $94.93(30.07)$ \\
\hline TMT Part B-A, mean (SD) ${ }^{\dagger}$ & $79.40(43.57)$ & $56.93(20.75)$ \\
\hline
\end{tabular}

*significant difference with $\mathrm{p}<0.05,{ }^{\dagger}$ significant difference with $\mathrm{p}<0.01$. RAVLT: Rey Auditory Verbal Learning Test, ROCF: Rey-Osterrieth complex figure, PM38: Raven's progressive matrices 1938, MCST: Modified Card Sorting Test, STROOP: Stroop colour word test, TMT: Trail Making Test, SD: standard deviation

B and TMT Part B-A showed a significant improvement at 6 months (T1) followed by a further significant improvement between 6 months and 1 year of abstinence (T2).

\section{Comparison of alcoholic performance throughout 1-year follow-up versus control data}

In order to investigate whether the alcoholic group recovered cognitive performance after 6-months and 1-year abstinence, we compared their subtest scores at T1 and T2 with control group (Table 4). Firstly, no statistical significant differences were found between the alcoholics at T1 and T2 follow-up and control subjects respect to demographic and clinical variables (also for HAMD scores), thus the two groups were still well matched.

With respect to comparison between $\mathrm{T} 1$ and control group, t-test results showed that verbal memory and visuospatial abilities (excepted Copy of Figures) still seemed compromised because the subtests scores were significant. Conversely, yet at six months executive functions seemed to be recovered because no statistical significant differences were found in comparison to the control group.

With respect to comparison between $\mathrm{T} 2$ and control group, t-test results showed that verbal memory was still compromised because the subtests scores of control group were statistically higher than alcoholics at 1-year abstinence. Visuospatial abilities seem to be in part recovered because, although the scores of control group were statistically higher than alcoholics at 1-year abstinence for ROCF-IR and PM38, no significant differences in other subtests were found. Finally, at 1-year, abstinent alcoholic group seemed to recover executive functions, because we did not find significant differences in comparison to the control group.

\section{DISCUSSION}

Patients compared to healthy controls performed worse in all cognitive domains investigated at baseline. Specifically, we found alterations in both short and long term verbal memory, in praxic-constructive and graph-motor skills, in both short and long term visual-spatial memory, in executive functions and more generally in fluid and general non-verbal intelligence. The existence of functional deficits in all investigated cognitive domains, is in line with the picture of a widespread deficit in extended brain networks that connect different brain structures. $^{19}$

These results also confirm some data in the literature to support the presence of a dysexecutive syndrome in subjects with alcohol dependence. In particular, in the study of Ratti et al., ${ }^{10}$ the alcoholics were found to be impaired in a wide range of executive domains, with the exception of the Stroop test which nevertheless showed a trend towards statistically significant differences. However, the performance at the Stroop test of our alcoholics sample, was strongly affected by "interference effects," showing the inability of the patients, compared with controls, to inhibit automatic responses and maintaining sustained attention to the ongoing task. Of note, we found a 
Table 4. Repeated measures ANOVA for alcoholic group; unpaired $t$ tests between $\mathrm{T} 1, \mathrm{~T} 2$ alcoholic group and data from control group

\begin{tabular}{|c|c|c|c|c|c|c|c|c|c|}
\hline & \multicolumn{5}{|c|}{ Alcoholic group } & \multirow{2}{*}{$\begin{array}{l}\text { Tuckey's } \\
\text { post hoc }\end{array}$} & \multirow{2}{*}{$\begin{array}{c}\begin{array}{c}\text { Control } \\
\text { group }\end{array} \\
\text { Single measure } \\
\text { M (SD) }\end{array}$} & \multirow{2}{*}{$\begin{array}{c}\text { Control } \\
\text { group vs. } \\
\mathrm{T} 1 \\
\mathrm{t}\end{array}$} & \multirow{2}{*}{$\begin{array}{c}\text { Control } \\
\text { group vs. } \\
\mathrm{T} 2 \\
\mathrm{t}\end{array}$} \\
\hline & $\begin{array}{c}\text { T0 } \\
\mathrm{M}(\mathrm{SD})\end{array}$ & $\begin{array}{c}\mathrm{T} 1 \\
\mathrm{M}(\mathrm{SD})\end{array}$ & $\begin{array}{c}\mathrm{T} 2 \\
\mathrm{M}(\mathrm{SD})\end{array}$ & $\mathrm{F}$ & $\eta^{2}$ & & & & \\
\hline \multicolumn{10}{|l|}{ Craving } \\
\hline OCDS obsessive & $7.74(4.06)$ & $4.59(2.97)$ & $1.70(1.54)$ & $103.61^{\dagger}$ & 0.40 & $\mathrm{~T} 0>\mathrm{T} 1>\mathrm{T} 2$ & - & - & - \\
\hline OCDS compulsive & $9.30(4.07)$ & $4.70(3.37)$ & $1.26(1.23)$ & $119.38^{\dagger}$ & 0.52 & $\mathrm{~T} 0>\mathrm{T} 1>\mathrm{T} 2$ & - & - & - \\
\hline \multicolumn{10}{|l|}{ Verbal memory } \\
\hline RAVLT_IR & $38.91(9.41)$ & $41.19(8.65)$ & $41.38(8.04)$ & $822.35^{\dagger}$ & 0.02 & $\mathrm{~T} 0<\mathrm{T} 1=\mathrm{T} 2$ & $49.83(8.33)$ & $4.52^{\dagger}$ & $4.13^{+}$ \\
\hline RAVLT_DR & $8.42(2.80)$ & $7.76(2.98)$ & $8.49(2.81)$ & $254.81^{\dagger}$ & 0.01 & $\mathrm{~T} 0>\mathrm{T} 1<\mathrm{T} 2$ & $10.10(2.25)$ & $3.82^{\dagger}$ & $2.59^{*}$ \\
\hline \multicolumn{10}{|l|}{ Visuospatial abilities } \\
\hline ROCF_IR & $27.61(6.81)$ & $28.79(6.40)$ & $29.08(6.57)$ & $573.27^{\dagger}$ & 0.00 & $\mathrm{~T} 0<\mathrm{T} 1<\mathrm{T} 2$ & $31.33(1.87)$ & $2.82^{\dagger}$ & $2.05^{*}$ \\
\hline ROCF_DR & $9.32(6.42)$ & $13.86(7.37)$ & $16.69(6.26)$ & $127.43^{\dagger}$ & 0.17 & $\mathrm{~T} 0<\mathrm{T} 1<\mathrm{T} 2$ & $16.47(5.87)$ & $2.01^{*}$ & -0.15 \\
\hline PM38 & $31.24(10.42)$ & $34.07(9.66)$ & $36.09(9.75)$ & $337.87^{\dagger}$ & 0.04 & $\mathrm{~T} 0<\mathrm{T} 1<\mathrm{T} 2$ & $40.91(3.89)$ & $4.23^{\dagger}$ & $2.82^{*}$ \\
\hline Copy of figures & $8.29(2.52)$ & $8.69(2.03)$ & $9.41(1.72)$ & $522.08^{\dagger}$ & 0.05 & $\mathrm{~T} 0<\mathrm{T} 1<\mathrm{T} 2$ & $9.29(1.42)$ & 1.70 & -0.32 \\
\hline \multicolumn{10}{|l|}{ Executive functions } \\
\hline MCST_CAT & $4.67(1.69)$ & $4.93(1.57)$ & $4.89(1.28)$ & $380.67^{\dagger}$ & 0.01 & $\mathrm{~T} 0<\mathrm{T} 1=\mathrm{T} 2$ & $5.18(1.38)$ & 1.88 & 0.86 \\
\hline MCST_EP & $3.87(4.76)$ & $2.33(3.14)$ & $2.28(2.25)$ & $23.88^{\dagger}$ & 0.04 & $\mathrm{~T} 0>\mathrm{T} 1=\mathrm{T} 2$ & $2.09(3.40)$ & -1.56 & -0.26 \\
\hline STROOP_EI & $1.76(3.10)$ & $0.89(1.66)$ & $0.76(1.31)$ & $10.11^{\dagger}$ & 0.04 & $\mathrm{~T} 0>\mathrm{T} 1>\mathrm{T} 2$ & $0.73(0.50)$ & -1.10 & -0.15 \\
\hline STROOP_TI & $24.65(9.23)$ & $20.16(8.97)$ & $19.27(7.18)$ & $237.66^{\dagger}$ & 0.07 & $\mathrm{~T} 0>\mathrm{T} 1>\mathrm{T} 2$ & $19.02(5.42)$ & -0.53 & -0.16 \\
\hline TMT_B & $123.78(50.01)$ & $101.74(33.04)$ & $95.02(37.41)$ & $255.55^{\dagger}$ & 0.08 & $\mathrm{~T} 0>\mathrm{T} 1>\mathrm{T} 2$ & $94.93(30.07)$ & -0.63 & -0.01 \\
\hline TMT_BA & $88.29(44.71)$ & $70.48(31.04)$ & $58.82(34.77)$ & $143.83^{\dagger}$ & 0.10 & $\mathrm{~T} 0>\mathrm{T} 1>\mathrm{T} 2$ & $56.93(20.75)$ & -1.55 & -0.28 \\
\hline
\end{tabular}

T0: baseline, T1: 6 months, T2: 1 year, <: that mean is significantly lower, =: that there is not significant differences between means, $>$ : that mean is significantly higher. *significant difference with $\mathrm{p}<0.05$, ${ }^{\dagger}$ significant difference with $\mathrm{p}<0.01$. OCDS: Obsessive-Compulsive Drinking Scale, RAVLT_IR: Rey Auditory Verbal Learning Test Immediate Recall, RAVLT_DR: Rey Auditory Verbal Learning Test Delayed Recall, ROCF_IR: Rey-Osterrieth complex figure Immediate Recall, ROCF_DR: Rey-Osterrieth complex figure Delayed Recall, PM38: Raven's progressive matrices 1938, MCST_CAT: Modified Card Sorting Test number of categories completed, MCST_EP: Modified Card Sorting Test number of perseverative errors, STROOP_EI: Stroop colour word test error interference effect, STROOP_TI: Stroop colour word test time interference effect, TMT_B: Trail Making Test Part B, TMT_BA: Trail Making Test Part B-A, SD: standard deviation

more general deficit in cognitive processing speed highlighted by the increase in execution times for all time tests (e.g. Stroop test, TMT).

The deficits of perceptual-motor and visual-spatial functions shown by our patients are consistent with some studies that support the hypothesis of the lateralization of brain damage in alcoholism. This is found in all those tasks where the right brain hemisphere is dominant, such as the visual learning and visuospatial abilities. ${ }^{13-16}$

Concerning the gender differences in alcohol-induced neurotoxicity and brain damage we have found that at baseline women performed worse that men in the domain of executive functions and in particular at Stroop interference errors. These data are in accordance with some evidence in the literature that shown that women are more vulnerable that men to the toxic effects of alcohol. ${ }^{36}$

Regarding the duration of abstinence, the results of our study clearly show that abstinence from alcohol promotes the recovery of cognitive functions. In fact, after six months of stopping alcohol consumption, patients showed a significant improvement in cognitive functions, this recovery then increased all over the year of abstinence. Of note, our sample of abstinent alcoholics showed a worse performance on long term verbal memory task (RAVLT-DR) after 6 months of sobriety respect to baseline followed by a recovery after 1 year of abstention. This is in line with previous studies that shown an impairment of verbal memory in short term abstinence. ${ }^{19}$ Patients showed a significant improvement both in attentional and set-shifting capacity and in praxic-constructive skills and visual-spatial learning. Interestingly, some studies reported that alcoholics in short-term abstinence performed worse than healthy controls in tasks of visual-spatial memory and that visual-spatial abilities are recovered only after a more prolonged abstinence. ${ }^{37}$ This is also the case of our sample of alcohol dependent patients. In fact, if we consider the clinical cut-off scoring of the ROCF-IR test, the baseline performance 
of our alcoholics subjects showed a deficit comparable to patients with focal neurological damage. Therefore a slower recovery of the visual-spatial abilities could be explained by the great difficulty of patients in performing this specific kind of tasks. In fact, we found a recovery of the performance above the clinical cut-off after one year of abstinence from alcohol, which at six months showed a trend towards improvement. We also found that our alcoholic subjects recovered in the TMT part B and B-A which emphasizes the great improvement of set-shifting skills probably due to phenomena of neuronal plasticity induced by alcohol consumption suspension. ${ }^{1}$ This was confirmed when the performance of the clinical sample, taken one year after the suspension of alcohol consumption, was compared with control subjects. In fact, the performance of the two experimental groups has become similar, except for verbal memory and measures of visuospatial skills and general non-verbal intelligence. For this reason, we can rule out the role of practice effects in the T0-T2 comparison because patients still showed poorer performance respect to the control data in those tests for which alternate forms were not available. Thus, it is reasonable that the improvement at 1-year follow-up is mainly due to the abstinence and not to the practice effects of repeated assessments.

Another interesting finding concerns the improvement in tasks execution times, highlighted by increased performance in all the tests that take into account the time variable. This would show how the cognitive processing speed of alcoholics patients is severely tested by the encounter with alcohol, making alcoholics slower than healthy controls in all time tests. In clinical practice, the detection of test execution slowness is a clear signal that identifies the ethanol neurotoxicity in progress.

Interestingly, although our study does not provide any conclusive data about the relationship between treatment and cognition, these preliminary findings seem to suggest a positive effect of anti-craving medications on the cognitive performance of alcoholics. In order to improve the personalization of care, further studies should investigate the long term effects of different anti craving drugs on different cognitive domains, particularly on those that do not achieve the score of control data after a year of abstention (i.e. verbal memory, visuospatial abilities and general non-verbal intelligence).

Therefore, it is possible to state that alcohol is a highly toxic substance that adversely affects the general cognitive functioning, both in terms of accuracy of the response during the tasks and in terms of execution speed. The daily life of alcohol dependent subjects is negatively affected because of their lower cognitive performance. Moreover this cognitive impairment maintains the addiction because skills related to the prefrontal cortex such as problem solving, learning from experience and cognitive flexibility are skills that if altered pro- mote the condition of addiction itself by increasing the risk of abuse and relapse in additive behaviour. In line with previous evidence, it can be speculated that alcoholism result from the imbalance between two systems (i.e. hyperactive impulsive system and hypoactive reflective' one). ${ }^{38-40}$ Due to the failure of the executive functions, alcoholics could not learn by the negative experience of alcohol consumption, or by adversative experience induced by drugs such as disulfiram. Furthermore problem solving and cognitive flexibility deficits place these patients in a less favourable condition for being able to positively deal with the addiction behaviour, just in terms of poorer cognitive resources that they can spend to get out of the addiction.

There were some limitations to this study, including the relatively small sample size for a longitudinal study and the lack of comorbidity. It is also possible that we have dealt with a highly specific sample due the fact that patients and controls did not differ in some demographic variables such as education level and rate of subjects employed. The lack of comorbidity alongside a good social functioning (i.e. employment is maintained) could explain the relatively low rate of drop out at 1 year follow-up. Even if the subject seems to have "mild" form of AUD on average and no severe cognitive deficit would be expected in these population (i.e. employment is maintained) we have found that alcoholics subjects performed worse than healthy control and this suggest a direct effect of alcohol on the cognitive functions.

Moreover we have not addressed the relationships between age and drinking history variables, as well as cognitive performances. Finally, we have not perform an imaging study to detect some cerebral changes in the follow up period and further study are encouraged to perform it.

However, the findings of studies like this are valuable mainly because they offer interesting insights for clinical practice. In particular, the need to include a cognitive screening during the anamnesis process is clear and during the clinical evaluation of patients with alcohol dependence, to assess the cognitive functioning and developing appropriate therapeutic strategies that take into account the resources and cognitive weaknesses of each person who seeks treatment. Beyond the possible positive effects of anti-craving drugs, patients would also beneficiate from cognitive training which might potentially reduce the number of relapsers and improve cognitive functions. ${ }^{41-43}$ Developing a profitable intervention plan for the patient also means measuring the cognitive domains of the subject to improve the understanding of the clinical characteristics of the patient and at the same time structuring personalized plans to avoid waste of resources and improving the quality of life of these patients and their compliance to treatment. 


\section{REFERENCES}

1. Fein G, Cardenas VA. Neuroplasticity in human alcoholism: studies of extended abstinence with potential treatment implications. Alcohol Res 2015;37:125-141.

2. Ioime L, Guglielmo R, Kadilli I, Janiri L. The importance of cognitive assessment in the management of psychiatric disorders. Res Adv Psychiatry 2014;1:44-45.

3. Zaninotto L, Solmi M, Veronese N, Guglielmo R, Ioime L, Camardese $\mathrm{G}$, et al. A meta-analysis of cognitive performance in melancholic versus non-melancholic unipolar depression. J Affect Disord 2016;201:15-24.

4. Zaninotto L, Guglielmo R, Calati R, Ioime L, Camardese G, Janiri L, et al. Cognitive markers of psychotic unipolar depression: a meta-analytic study. J Affect Disord 2015;174:580-588.

5. Allsop S, Saunders B, Phillips M. The process of relapse in severely dependent male problem drinkers. Addiction 2000;95:95-106.

6. Wolwer W, Burtscheidt W, Redner C, Schwarz R, Gaebel W. Out-patient behaviour therapy in alcoholism: impact of personality disorders and cognitive impairments. Acta Psychiatr Scand 2001;103:30-37.

7. Camchong J, Stenger A, Fein G. Resting-state synchrony in long-term abstinent alcoholics. Alcohol Clin Exp Res 2013;37:75-85.

8. Bush G, Luu P, Posner MI. Cognitive and emotional influences in anterior cingulate cortex. Trends Cogn Sci 2000;4:215-222.

9. Bolla KI, Funderburk FR, Cadet JL. Differential effects of cocaine and cocaine alcohol on neurocognitive performance. Neurology 2000;54: 2285-2292.

10. Ratti MT, Bo P, Giardini A, Soragna D. Chronic alcoholism and the frontal lobe: which executive functions are imparied? Acta Neurol Scand 2002;105:276-281.

11. Saraswat N, Ranjan S, Ram D. Set-shifting and selective attentional impairment in alcoholism and its relation with drinking variables. Indian J Psychiatry 2006;48:47-51.

12. Loeber S, Duka T, Welzel H, Nakovics H, Heinz A, Flor H, et al. Impairment of cognitive abilities and decision making after chronic use of alcohol: the impact of multiple detoxifications. Alcohol Alcohol 2009;44:372-381.

13. Fein G, Bachman L, Fisher S, Davenport L. Cognitive impairments in abstinent alcoholics. West J Med 1990;152:531-537.

14. Lezak MD. Neuropsychological Assessment. New York: Oxford University Press; 1995.

15. Oscar-Berman M, Schendan HE. Asymmetries of Brain Function in Alcoholism: Relationship to Aging. In: Connor LT, Obler LK, Editors. Neurobehavior of Language and Cognition. New York: Springer US, 2002, p.213-240.

16. Wilson JT, Wiedmann KD, Phillips WA, Brooks DN. Visual event perception in alcoholics. J Clin Exp Neuropsychol 1988;10:222-234.

17. Kopera M, Wojnar M, Brower K, Glass J, Nowosad I, Gmaj B, et al. Cognitive functions in abstinent alcohol-dependent patients. Alcohol 2012;46:665-671.

18. Glass JM, Buu A, Adams KM, Nigg JT, Puttler LI, Jester JM, et al. Effects of alcoholism severity and smoking on executive neurocognitive function. Addiction 2009;104:38-48.

19. Stavro K, Pelletier J, Potvin S. Widespread and sustained cognitive deficits in alcoholism: a meta-analysis. Addict Biol 2013;18:203-213.

20. American Psychiatric Association. American Psychiatric Association. Task Force on DSM-IV. Diagnostic and Statistical Manual of Mental Disorders: DSM-IV-TR. Washington, DC: American Psychiatric Association; 2000.

21. First MB. Structured Clinical Interview for DSM-IV Axis I Disorders: SCID-I: Clinician Version: Administration Booklet. Washington, DC: American Psychiatric Press; 1997.
22. Martinotti G. Pregabalin in clinical psychiatry and addiction: pros and cons. Expert Opin Investig Drugs 2012;21:1243-1245.

23. Guglielmo R, Ioime L, Solaroli S, Janiri L. Pharmacological treatments in alcohol use disorders: state of art and new perspectives. Clin Ter 2015;166:262-270.

24. Sobell L, Sobell M. Alcohol Timeline Followback User's Manual. Toronto: Addiction Research Foundation; 1995.

25. Guy W. ECDEU Assessment Manual for Psychopharmacology. Rockville: U.S. Department of Health, Education, and Welfare; 1976.

26. Janiri L, Calvosa F, Dario T, Pozzi G, Ruggeri A, Addolorato G, et al. The Italian version of the Obsessive-Compulsive Drinking Scale: validation, comparison with the other versions, and difference between type 1- and type 2-like alcoholics. Drug Alcohol Depend 2004;74:187195.

27. Hamilton M. A rating scale for depression. J Neurol Neurosurg Psychiatry 1960;23:56-62.

28. Caffarra P, Vezzadini G, Zonato F, Copelli S, Venneri A. A normative study of a shorter version of Raven's progressive matrices 1938. Neurol Sci 2003;24:336-339.

29. Giovagnoli AR, Del Pesce M, Mascheroni S, Simoncelli M, Laiacona M, Capitani E. Trail making test: normative values from 287 normal adult controls. Ital J Neurol Sci 1996;17:305-309.

30. Caffarra P, Vezzadini G, Dieci F, Zonato F, Venneri A. Una versione abbreviata del test di Stroop: dati normativi nella popolazione italiana. Nuova Rivista di Neurologia 2002;12:111-115.

31. Caffarra P, Vezzadini G, Dieci F, Zonato F, Venneri A. Modified Card Sorting Test: normative data. J Clin Exp Neuropsychol 2004;26:246-250.

32. Carlesimo GA, Caltagirone C, Fadda L, Marfia L, Gainotti G, Gallassi $\mathrm{R}$, et al. The Mental Deterioration Battery (Part 3): standardization and diagnostic reliability in the identification of demented patients. Archivio di psicologia, neurologia e psichiatria 1995;56:489-502.

33. Caffarra P, Vezzadini G, Dieci F, Zonato F Venneri A. Rey-Osterrieth complex figure: normative values in an Italian population sample. Neurol Sci 2002;22:443-447.

34. Carlesimo GA, Caltagirone C, Fadda L, Marfia L, Gainotti G, Gallassi $\mathrm{R}$, et al. The Mental Deterioration Battery (Part 2): standardization and diagnostic reliability in the identification of demented patients. Archivio di psicologia, neurologia e psichiatria 1995;56:471-488.

35. Cohen J. Statistical Power Analysis for the Behavioral Sciences. Hillsdale, NJ: L. Erlbaum Associates; 1988.

36. Hommer D, Momenan R, Kaiser E, Rawlings R. Evidence for a gender-related effect of alcoholism on brain volumes. Am J Psychiatry 2001;158:198-204.

37. Leber WR, Jenkins RL, Parsons OA. Recovery of visual-spatial learning and memory in chronic alcoholics. J Clin Psychol 1981;37:192-197.

38. Camchong J, Endres M, Fein G. Decision making, risky behavior, and alcoholism. Handb Clin Neurol 2014;125:227-236.

39. Field M, Wiers RW, Christiansen P, Fillmore MT, Verster JC. Acute alcohol effects on inhibitory control and implicit cognition: implications for loss of control over drinking. Alcohol Clin Exp Res 2010;34:1346-1352.

40. Moselhy HF, Georgiou G, Kahn A. Frontal lobe changes in alcoholism: a review of the literature. Alcohol Alcohol 2001;36:357-368.

41. Houben K, Nederkoorn C, Wiers RW, Jansen A. Resisting temptation: decreasing alcohol-related affect and drinking behavior by training response inhibition. Drug Alcohol Depend 2011;116:132-136.

42. Jones A, Field M. The effects of cue-specific inhibition training on alcohol consumption in heavy social drinkers. Exp Clin Psychopharmacol 2013;21:8-16.

43. Wiers RW, Gladwin TE, Rinck M. Should we train alcohol-dependent patients to avoid alcohol? Front Psychiatry 2013;4:33. 